\title{
A Survey on Deep Transfer Learning and Edge Computing for Mitigating the COVID-19
}

This paper was downloaded from TechRxiv (https://www.techrxiv.org).

\section{LICENSE}

CC BY 4.0

SUBMISSION DATE / POSTED DATE

22-06-2020 / 24-06-2020

\section{CITATION}

Sufian, Abu; Ghosh, Anirudha; Sadiq, Ali Safaa; Smarandache, Florentin (2020): A Survey on Deep Transfer Learning and Edge Computing for Mitigating the COVID-19. TechRxiv. Preprint.

https://doi.org/10.36227/techrxiv.12535010.v1

$\mathrm{DOI}$

10.36227/techrxiv.12535010.v1 


\title{
A Survey on Deep Transfer Learning and Edge Computing for Mitigating the COVID-19
}

\author{
Abu Sufian ${ }^{\mathrm{a}, *}$, Anirudha Ghosh ${ }^{\mathrm{a}}$, Ali Safaa Sadiq ${ }^{\mathrm{b}}$, Florentin Smarandache ${ }^{\mathrm{c}}$ \\ ${ }^{a}$ Department of Computer Science, University of Gour Banga, India \\ ${ }^{b}$ School of Maths. and Comp. Science, University of Wolverhampton, United of Kingdom \\ ${ }^{c}$ Mathematics Department, University of New Mexico, United States
}

\begin{abstract}
Global Health sometimes faces pandemics as are currently facing COVID-19 disease. The spreading and infection factors of this disease are very high. A huge number of people from most of the countries are infected within six months from its first report of appearance and it keeps spreading. The required systems are not ready up to some stages for any pandemic; therefore, mitigation with existing capacity becomes necessary. On the other hand, modern-era largely depends on Artificial Intelligence(AI) including Data Science; Deep Learning(DL) is one of the current flag-bearer of these techniques. It could use to mitigate COVID-19 like pandemics in terms of stop spread, diagnosis of the disease, drug \& vaccine discovery, treatment, and many more. But this DL requires large datasets as well as powerful computing resources. A shortage of reliable datasets of a running pandemic is a common phenomenon. So, Deep Transfer Learning(DTL) would be effective as it learns from one task and could work on another task. In addition, Edge Devices(ED) such as IoT, Webcam, Drone, Intelligent Medical Equipment, Robot, etc. are very useful in a pandemic situation. These types of equipment make the infrastructures sophisticated and automated which helps to cope with an outbreak. But these are equipped with low computing resources, so, applying DL is also a bit challenging; therefore, DTL also would be effective there. This article scholarly studies the potentiality and challenges of these issues. It has described relevant technical backgrounds and reviews of the related recent state-of-the-art. This article also draws a pipeline of DTL over Edge Computing as a future scope to assist the mitigation of any pandemic.
\end{abstract}

Keywords: AI for Good, COVID-19, Deep Learning, Edge Computing, Pandemic, Review, Transfer Learning.

\footnotetext{
*Corresponding author

Email address: sufian.csa@gmail.com (Abu Sufian)
} 


\section{Introduction}

The COVID-19 is a disease caused by a novel coronavirus called 'SARSCoV-2'. This virus is transferable from human to human and its spreading, and infection factors are very high [1, 2, Over nine million people are infected and approx half million are died within just six months from it's fist report, and it is increasing steadily 1 . The World Health Organization (WHO) has declared it a pandemic [3, 4]. But this is not the only pandemic human civilization is facing, there are many outbreaks had come in the past or it may come in the future [5, 6]. The appropriate drugs, vaccines, infrastructure, etc. are not available up to some stages of any outbreaks. Therefore, mitigate these types of diseases with existing capacity becomes most important in those stages [7, 8]. Many researchers from all over the world trying hard to develop such kind of techniques to cope with such challenges [9, 10, 11].

Modern-era largely depends on Artificial Intelligence(AI) including Data Science; Deep Learning (DL) is one of the current flag-bearer of these techniques [12]. Therefore, these techniques could also assist to mitigate COVID19 like pandemics in terms of stop spread, diagnosis of the disease, drug \& vaccine discovery, treatment, and many more [13, 14]. But to trained this DL, large datasets as well as powerful computing resources are required. For a new pandemic, data insufficiency and it's variation over different geographic regions is a huge problem, so here Deep Transfer Learning (DTL) would be effective as it learns from one task and could apply in another task after required fine-tuning [15]. On the other hand edge devices such as IoT, Webcam, Drone, Intelligent Medical Equipment, Robot, etc. are very useful in any pandemic situation. These types of equipment make the infrastructures sophisticated and automated which helps to cope with an outbreaks [16]. Though, such devices are equipped with low computing resources which represent the main challenges of Edge Computing(EC) [17]. As a way to overcome this challenge, transfer learning could be a possible way to consolidate the needed computational power and facilitate more efficient EC. Therefore, DTL in edge devices as an EC could be smart techniques to mitigate a new pandemic [18]. This survey article has tried to report all these issues scholarly as potentialities and challenges with relevant technical backgrounds. Here, we also proposed a possible pipeline architecture for future scopes to brings DTL over EC to assists mitigation in any outbreaks.

\footnotetext{
${ }^{1}$ https://www.worldometers.info/coronavirus/
} 


\subsection{Contributions of this Article}

Some highlights of the contributions of this article are as follows:

- Presented a systematic study of Deep Learning (DL), Deep Transfer Learning(DTL) and Edge Computing(EC) to mitigate COVID-19.

- Surveyed on existing DL, DTL, EC, and Dataset to mitigate pandemics with potentialities and challenges.

- Drawn a precedent pipeline model of DTL over EC for a future scope to mitigate any outbreaks.

- Given brief analyses and challenges wherever relevant in perspective of COVID-19.

\subsection{Organization of the Article}

Starting from the introduction in section 1, the remainder of the article organized follows. Section 2 for technical background whereas review of generic state-of-the-art of DTL in EC in section 3. Existing computing(DL, DTL, EC \& Dataset) to mitigate pandemic in section 4 . A proposed pipeline of DTL in EC to mitigate pandemics in section 5, Finally, conclusion in section 6 .

\section{Technical Backgrounds}

The main focus of this article is how DL, DTL, EC, and it's associate could assist to mitigate any pandemics. The possible roles and challenges of these techniques in a pandemic, especially for COVID-19, are mentioned in section 4. This section has tried to bring an overview and general progress of DL, DTL, and EC in the following three subsections.

\subsection{Deep Learning $(D L)$}

Deep learning (DL) (also known as hierarchical learning or deep structured learning) is one of the great inventions for modern-era of Artificial Intelligence (AI) [12]. Until the decade '90s, classical machine learning techniques were used for making inferences on data and prediction. Nevertheless it had several drawbacks such as depend on handcrafted features, bounded by human-level accuracy, etc [19]. But in DL, handcrafted feature engineering is not required rather features are extracted from data during training. In addition, DL can make more accurate classifications and predictions with the help of innovative algorithms, computing power of modern machines, and 
the availability of Big Datasets [20]. Nowadays, DL methods have been successfully applied for several AI-based medical applications such as Magnetic Resonance Imaging (MRI) images analysis for cancer and diabetes diagnoses, conjunction with biometric characteristics, etc [21].

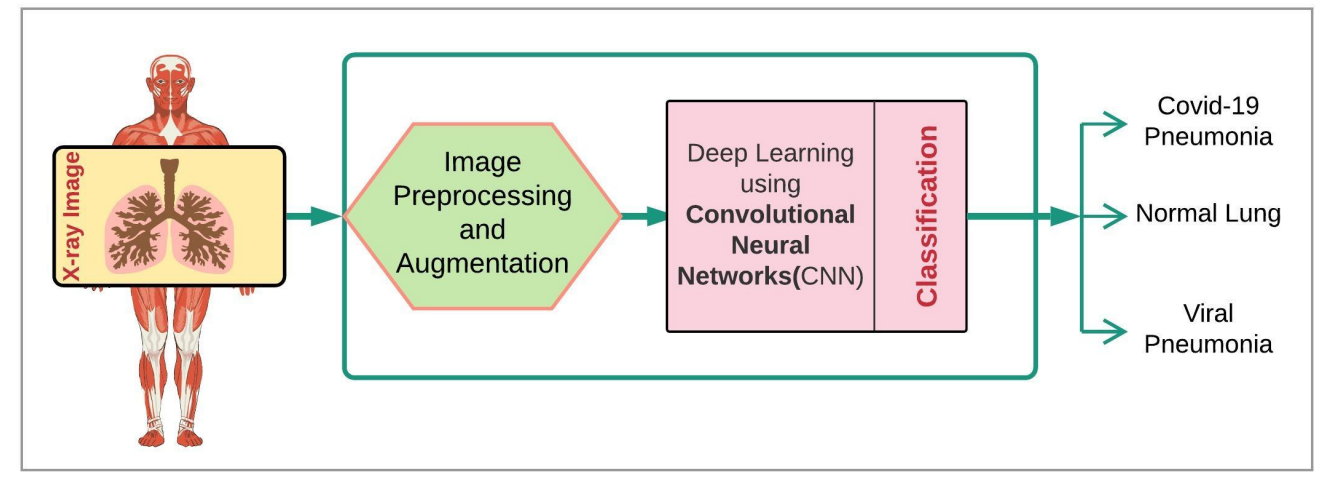

Figure 1: A overall block diagram of a Deep learning-based screening system.

DL is a kind of learning algorithm or model under the umbrella of AI which is based on Artificial Neural Networks(ANN) [22]. These models are trained using dataset through backpropagation algorithm [23] and a suitable optimizer method [24]. The inherent capacities of such DL model such massive parallelism, non-linearity, and capabilities of feature extraction have made them powerful and widely used [20]. There are several variety of DL algorithm such as Convolutional Neural Networks(CNN) [25, 26], Recurrent Neural Networks (RNN) [27], Long Short Term Memory(LSTM) [28], GAN [29], etc. After success of a CNN-based model, called AlexNet [30, many deep learning model has proposed such ZFNet [31], VGGNet [32], GooglNet [33], ResNet [34, DenseNet [35], etc specially for computer vision tasks [36]. In figure 1 we try to illustrate a typical methodology of a DL based screening system, where the system uses a DL algorithm (CNN) to predict whether the $\mathrm{X}$-ray images of suspected patient's lung is normal or having viral pneumonia or COVID-19 pneumonia.

In the time of the COVID-19 crisis, when the numbers of infected patients are at a time very high and the disease is still spreading, many research groups are using the DL techniques for screening COVID-19 patients by detection fever temperature, viral and COVID-19 pneumonia, etc. In addition, DL is using or could be used for other purposes such as patient care, detection systematic social distancing violation, etc [14]. As for reference, S. Wang et.al used a CNN-based DL for screening COVID-19 patients with an accuracy, sensitivity, and specificity of $89.5 \%, 87 \%$, and $88 \%$ respectively by using their computed tomography (CT) images [37]. Similarly, in another study [38] L. 
Wang et.al used chest X-ray images for a screening of COVID-19 cases with $83.5 \%$ accuracy. The description of such works is in section 4.1.1.

\subsection{Deep Transfer Learning(DTL)}

Transfer Learning is a technique that effectively uses knowledge of an already learned model to solve another new task (possibly related or little related) with require of minimal re-training or fine-tuning [39, 40]. Since DL requires a massive training data compared to traditional machine learning methods. So, the requirement of a large amount of labeled data is a big problem in solving some critical domain-specific tasks, specifically the applications for the medical domain, where the making of large-scale, high-quality annotated medical datasets is very complex, and expensive [41]. In addition, the usual DL model requires large computing power such as GPU enabled sever, although researchers are trying hard to optimizing it [42, 43]. Therefore, Deep Transfer Learning (DTL), a DL based Transfer Learning try to overcome this problem [44]. DTL significantly reduces the demand for training data and training time for a target domain-specific task by choosing a pretrained model (trained on another large dataset of same target domain) for a fixed feature extractor [45] or for further fine-tuning [46]. Figure 2 demon-

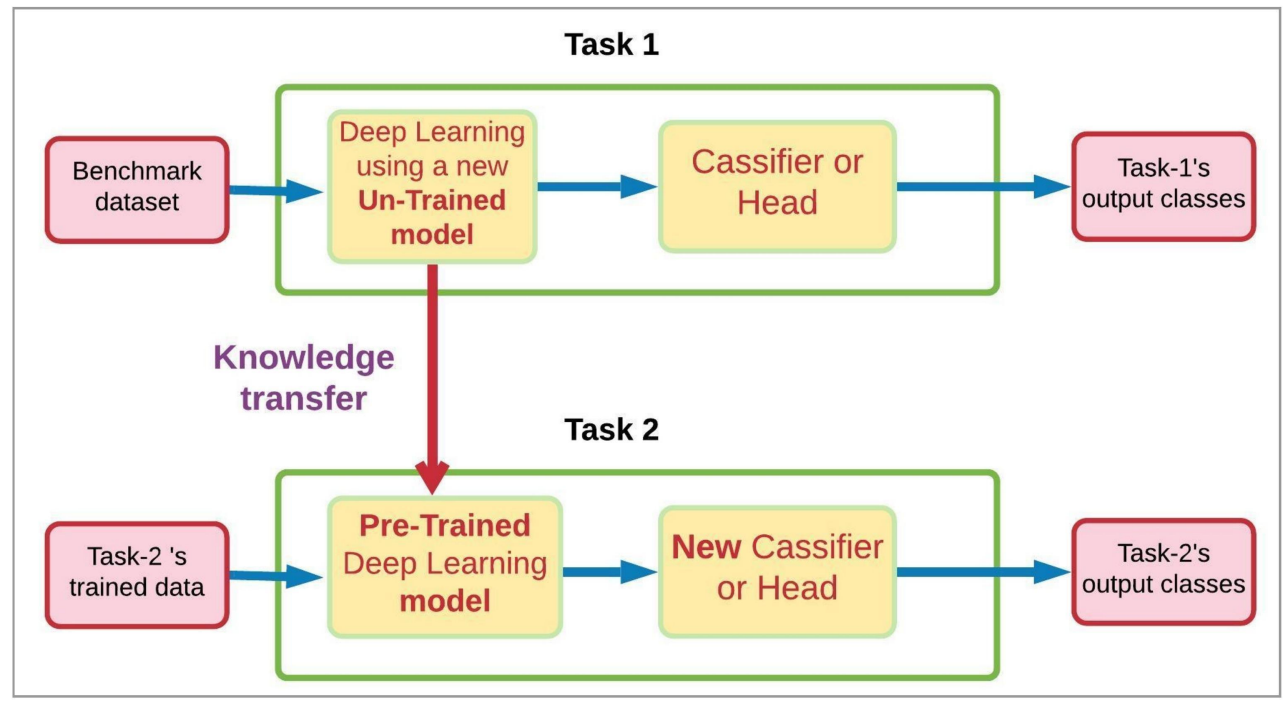

Figure 2: Block diagram of an example of Deep Transfer Learning.

strating the main steps methodology of a Deep Transfer Learning approach, where an untrained model is trained using benchmark dataset (task-1) for feature extraction. Then that pre-trained model is further used to tackle a new challenge such as the task (task-2) of COVID-19 by just replacing only few last layers in the head of the architecture and required fine-tuning. 
So far, many DTL models have been proposed [15]. A few recent are reported and discussed in the article. In a research study [44, Mingsheng Long et.al proposed a joint adaptation network. It learns a transfer network by aligning the joint distributions of multiple domain-specific layers across domains based on a joint maximum mean discrepancy. In another study [47, Yuqing Gao and Khalid M. Mosalam proposed a state-of-the-art transfer learning model based on VGG model [32. They have used ImagNet [48] dataset for features extractor and their hand label construction images for fine-tuning. Abnormality classification in MR images through DTL proposed in a study [49]. The authors of that study also have used pre-trained ResNet34 model with fine-tuning. In a research practice [50], a DTL for diagnosing faults in target applications without labeling was proposed. Their framework used condition distribution adaptation. Q-TRANSFER [51], another DTL framework proposed by Trung V. Phan et.al. To mitigate the dataset insufficiency problem in the context of communication networking, a DTL-based reinforcement learning approach is used.

As the COVID-19 disease spread is terrifying all over the world, screening, quarantine, and providing appropriate treatment to COVID-19 patients has become the first priority in the current scenario. But the global standard diagnostic pathogenic laboratory testing is massive time consuming and more costly with significant false-negative results [52]. At the same time, tests are are hardly to be taken place in the common healthcare centres or hospital due to limited resources and places compared with the high volume of cases at one time. To combat this kind of situation, the researcher from this domain are trying hard to develop some possible DTL models to mitigate this challenges [53, 54]. As for example M. Loey et.al in [53] use DTL along with the GAN model on their very limited, only 307 chest X-ray images to test COVID-19 disease based patient chest X-ray. Here, they have three pre-trained stateof-the-art model namely AlexNet [30, GoogleNet [33], and ResNet18 [34]. Among these three pre-trained GoogleNet give the highest accuracy in their experimental studies.

\subsection{Edge Computing (EC)}

In the era of cloud computing, maximum tech companies in the world rely on very few selected cloud providers for hosting and computation power. The user's data from millions of devices around the world is being delivered to some centralized cloud providers for processing or computation. This data transformation always resulted in extra latency and extra bandwidth consumption [55]. The explosive proliferation of IoT devices along with the requirement of real-time computing power have forced to move the scenario of computing paradigm towards Edge computing. Therefore, instead of relying 
on doing all the work at a cloud, it focuses to start the computational process close to the IoT devices (near to the source of data) in order to reduce the utilized bandwidth and latency [56, 57]. Sometime in Edge computing, an additional nearby server called Fog is associated between the cloud and the Edge or IoT devices. It locally stores the copy of densely used data from the cloud and it provides additional functionality to IoT devices to analyze and process their data locally with real-time working capability. Hence only the relevant data from IoT devices is need to transferred to the cloud through the Fog server [58].

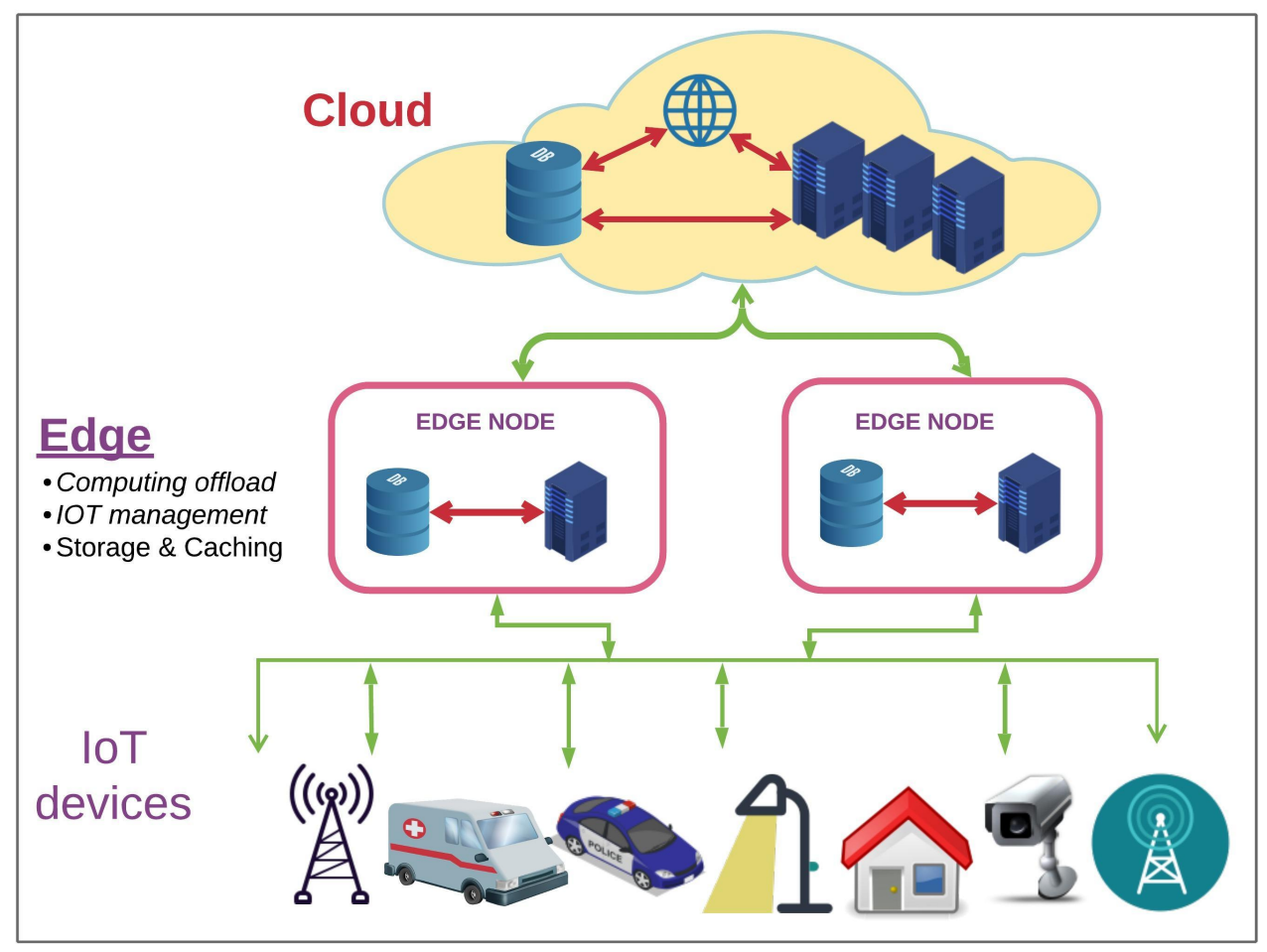

Figure 3: Hierarchy of Edge, Fog, and Cloud Computing.

In figure 3 the hierarchy of a possible framework for Edge Computing association with Fog and Cloud computing is illustrated. The data are collected from various IoT devices are being pre-processed before sending by Edge to Fog server for the analysis and computation with the real-time speed (because of the minimal distance between Edge layer and IoT devices and the local database of Fog). While the cloud holds the central control system and it manages the whole database of the system. The database on the cloud is continuously uploaded by the Fog only when it has important data or information. 
Although EC is not a new concept but it becomes popular in the last five years in the era of IoT [59, 60]. Few recent different type of state-of-the-art of $\mathrm{EC}$ are mentioned in this section as a way to familiarize the reader with the recent development with the era of $\mathrm{EC}$ and its potential benefits in mitigating COVID-19 as pandemic. EdgeIoT [61, a study of mobile EC proposed by X. Sun et.al. It is a SDN-based EC work with Fog Computing(FC) 62 to provide computational load locally. In a study [63], F. Wang et.al have proposed a joint offloading strategy of mobile EC and wireless power transfer. This scheme tried to address energy consumption, latency, and access point issues in IoT. In another study [64, Wei Ding et.al propose a field-programmable gate array-based depth-wise separable CNN accelerator to improve the system throughput and performance. They have used double-buffering-based memory channels to handle the data-flow between adjacent layers for mobile EC. On the other hand, G. Premsankar et.al in their case study [65] have discussed how efficient mobile gaming can run through EC. In a study [66], S. Wang et.al have proposed a mobile edge computing with an edge server placement strategy. In their multi-objective constraint optimization-based EC have tried reduced delay between a mobile user and an edge server. InEdge AI [66], an integrate the deep reinforcement learning techniques and Federated Learning framework with mobile edge systems are proposed by X. Wang et.al. This framework intelligently utilizes the collaboration among devices and edge nodes to exchange the learning parameters for betterment. In another recent study [67, an integrated two key technologies, ETSI and $3 \mathrm{GPP}$ are introduced to enhanced slicing capabilities to the edge of the $5 \mathrm{G}$ network. In the case of COVID-19 like pandemics, discussion of the possible role of EC is done in section 4.3 .

\section{Review of Related State-of-the-art}

Although the whole article is referred and cited current relevant stateof-the-art wherever relevant, this section is dedicated to provide a review on some of the very generic recent state-of-the-art works related to transfer learning approaches over edge computing. As mentioned in section 2.1, the progress of DL is very fast but when it comes to application in Edge or IoT devices then a huge gap is noticeable 68]. However, researchers are working hard to cope with the challenges, as results in many computing ideas, optimized model, as well as some computing accelerator devices, comes in picture [69, 70]. Deep Transfer Learning as mentioned in section 2.2 is one such area that is useful where the size of datasets is not sufficient [44]. This transfer learning is also useful where computing resources are not sufficient such as Edge or IoT devices [71. Since edge computing becomes popular in 
the last few years, so, we restricted this review to the last five years with chronological order.

Lorenzo Valerio et.al have studied the trade-off between accuracy and traffic load of computing in edge-based on transfer learning [72. They have suggested that sometimes the partial model needs to move across edge devices and data will stay at those edge devices and vice-versa. In a study [73], Tingting Hou et.al proposed a transfer learning approach in edge computing for proactive content caching. In their learningbased cooperative caching technique they have used a greedy algorithm for solving the problem of cache content placement. On the other hand, Junjue Wang et.al proposed a model 744 for live video analytic through drone using edge computing. They have used a transfer learning approach to formulate a pre-trained model to apply a few aerial view image classification. In another study [75], Ragini Sharma et.al proposed a teacher(large networks) student(small network at edge) model using transfer learning. The applied different transfer learning techniques of teacher-student with considering accuracy and convergence speed.

Qiong Chen et.al used a multitask transfer learning in their work [76]. In their data-driven cooperative task allocation scheme, they have used the concepts of the Knapsack problem to prioritized the tasks before transferring them for use in another task. In a study [77], Wen Sun et.al suggested an edge-cloud framework. Here, pre-trained networks used in their framework that are trained in the cloud. In other work, Rih-Teng Wu et.al proposed an edge computing strategy for autonomous robots [78. They have used CNN with pruning through the transfer learning technique. In their presented work, pr-trained VGG16 [32] and ResNet18 [34] are used for classification after fine-tuning. Cartel [79], a model of collaborative transfer learning approach for edge computing was proposed by Harshit Daga et.al. Here, they have created a model-sharing environment where a pre-trained model was adapted by each edge according to the needs. In a study [80], Yiqiang Chen et.al proposed a framework using Federated Transfer Learning for Wearable Healthcare (FedHealth). They have first performed data aggregation using federated learning and then created personalized models for each edge using transfer learning. OpenEI [81], an edge intelligence framework that was proposed by Xingzhou Zhang et.al. This framework with lightweight software equips with the edges as well as intelligent computing and data sharing capability.

In a research study [82, Changyang She et.al proposed a reliable low latency communication and edge computing system. They have adopted deep transfer learning in the architecture to fine-tune the pre-trained networks in non-stationary networks. This proposed work was designed for future 6G 
networks systems. On the other hand, Guangshun Li et.al proposed a task allocation load balancing strategy for edge computing [83. They have used the concept of transfer learning from cloud to intermediate node to edge. In another study [84, Gary White and Siobhan Clarke have proposed a deep transfer learning-based edge computing for urban intelligent systems. They have also used VGG16 pretrained network at edge devices and experimented to classify Dog vs. Cat images. MobileDA [85], a domain adaption framework in edge computing was proposed by Jianfei Yang et.al. Here, a teacher network was trained in a server and transfer knowledge or feature to student networks was implemented at the edge side. Their model was evaluated and obtained promising results on an IoT-based WiFi gesture recognition scenario. Davy Preuveneers et.al proposed a resource and performance trade-off strategy for a smart environment [86]. They have used a transfer learning model for less training efforts in smart edge devices. In their study, multiobjective optimization also was utilized to optimize the trade-off between computing resources uses and performances.

\section{Existing Computing (DL, DTL, EC \& Dataset) to Mitigate Pan- demic}

As mentioned in section 1, the appropriate drugs, vaccines, infrastructure are not ready up to some stages of any pandemic. Therefore, to cope with challenges existing knowledge, infrastructures, AI-based models could be exploited to mitigate such pandemic. This section tried to bring four insights of the discussion topics and their roles in mitigating pandemics. Each of them is systematically discussed with potentiality with recent state-of-the-art and challenges.

\subsection{Deep Learning Approaches to Mitigate Pandemic}

\subsubsection{Potentiality}

As described in section 2.1, Deep Learning(DL) can extract features directly from labeled data. In COVID-19 like pandemic data are new, so, handcrafted feature engineering might be difficult. But for DL, no feature engineering required, so that problem could be solved. The DL can assist in many ways to mitigate COVID-19 like pandemics along with other healthcare issues [87]. Some of them are Testing Sample Classification, Medical Image Understanding, Forecasting, etc [88]. Some recent DL based models have already proposed to cope with pandemics are listed and their main features are highlighted in table 1. This table brings some proposed peer-reviewed as well as few promising pre-print works. Table 1 has placed some recent works in upper rows. 
Table 1: Recent DL based works to mitigate pandemics

\begin{tabular}{|c|c|c|}
\hline $\begin{array}{l}\text { Reference } \\
\text { of Proposed } \\
\text { Works }\end{array}$ & $\begin{array}{l}\text { Dedicated task } \\
\text { of a Pandemic }\end{array}$ & Main Contributions \\
\hline $\begin{array}{l}\text { F.Ucar and } \\
\text { D.Korkmaz } \\
89\end{array}$ & $\begin{array}{l}\text { Deep Bayes-SqueezeNet } \\
\text { based diagnosis of } \\
\text { COVID-19 from X-ray } \\
\text { images. }\end{array}$ & $\begin{array}{l}\text { - Develop an intelligent diagnosis system for COVID-19 } \\
\text { using practical DL networks for medical image processing. } \\
\text { - A new decision-making system for COVID-19 with the } \\
\text { integration of conventional and state-of-the-art methods } \\
\text { for chest X-ray images. }\end{array}$ \\
\hline $\begin{array}{l}\text { C.Butt et.al } \\
\text { 90] }\end{array}$ & $\begin{array}{l}\text { Screen coronavirus dis- } \\
\text { ease } 2019 \text { pneumonia. }\end{array}$ & $\begin{array}{l}\text { - A study that compared multiple CNN models to classify } \\
\text { CT samples with COVID-19, influenza viral pneumonia, } \\
\text { and no-infection. } \\
\text { - Shown an accuracy of } 0.996 \text { ( } 95 \% \text { CI: } 0.9891 .00) \text { for } \\
\text { COVID-19 vs Non-COVID-19 cases per CT studies, and } \\
\text { calculated a sensitivity: } 98.2 \% \text { and specificity: } 92.2 \% \text {. }\end{array}$ \\
\hline $\begin{array}{l}\text { S.Hu et.al } \\
91\end{array}$ & $\begin{array}{l}\text { COVID-19 Infection De- } \\
\text { tection and Classifica- } \\
\text { tion from CT Images. }\end{array}$ & $\begin{array}{l}\text { - Weakly supervised DL for detecting and classifying } \\
\text { COVID-19 infection from CT images. } \\
\text { - Minimize the requirements of labeling of CT images. }\end{array}$ \\
\hline $\begin{array}{l}\text { T.Ozturka, } \\
\text { et.al } 92\end{array}$ & $\begin{array}{l}\text { automated detection of } \\
\text { COVID-19 cases using } \\
\text { raw X-ray. }\end{array}$ & $\begin{array}{l}\text { - DL based COVID- } 19 \text { vs No-finding as well as COVID- } \\
19 \text { vs No-finding vs. Pneumonia as binary and multi- } \\
\text { classification model. } \\
\text { - A combined of YOLO and DarkNet model }[93 \text { used as } \\
\text { the backbone of the model to achieved a good accuracy. }\end{array}$ \\
\hline $\begin{array}{l}\text { E.Luz et.al } \\
{[94]}\end{array}$ & $\begin{array}{l}\text { COVID-19 Patterns De- } \\
\text { tection in X-ray Images. }\end{array}$ & $\begin{array}{l}\text { - Identification of COVID-19 disease. } \\
\text { - A resource efficient model with overall accuracy of } \\
91.4 \% \text {, COVID-19, sensitivity of } 90 \% \text { and positive pre- } \\
\text { diction of } 100 \% \text { in the dataset from } 95 \text {. }\end{array}$ \\
\hline $\begin{array}{l}\text { M. Zhou et.al } \\
96]\end{array}$ & $\begin{array}{l}\text { Differentiating novel } \\
\text { coronavirus and in- } \\
\text { fluenza pneumonias. }\end{array}$ & $\begin{array}{l}\text { - An early diagnosis tool on chest CT images for differen- } \\
\text { tiate Coronovirus pneumonia and normal Influenza with } \\
\text { transferability. }\end{array}$ \\
\hline $\begin{array}{l}\text { A. Lopez- } \\
\text { Rincon et.al } \\
97\end{array}$ & $\begin{array}{l}\text { Identification of SARS- } \\
\text { CoV-2 from Viral } \\
\text { Genome Sequences. }\end{array}$ & $\begin{array}{l}\text { - Interaction between viromics and DL. } \\
\text { - A DL-based model to develop an assisted detection tests } \\
\text { for SARS-CoV-2 }\end{array}$ \\
\hline $\begin{array}{l}\text { O.Gozes et.al } \\
98\end{array}$ & $\begin{array}{l}\text { Automated Detection \& } \\
\text { Patient Monitoring us- } \\
\text { ing Deep Learning and } \\
\text { CT Image Analysis. }\end{array}$ & $\begin{array}{l}\text { - A model utilizing } 2 \mathrm{D} \text { and } 3 \mathrm{D} \text { DL for clinical understand- } \\
\text { ing. } \\
\text { - Proposed a systematic continuous monitoring COVID- } \\
19 \text { patients and their clinical data to make a statistical } \\
\text { Corona score for monitoring their progress. }\end{array}$ \\
\hline $\begin{array}{l}\text { S.M. Ayy- } \\
\text { oubzadeh } \\
\text { et.al } 99 \text { ] }\end{array}$ & $\begin{array}{l}\text { Predict the incidence of } \\
\text { COVID-19 in Iran. }\end{array}$ & $\begin{array}{l}\text { - Data were mined from Google Trends website } \\
\text { - Linear regression and LSTM models were used to esti- } \\
\text { mate positive COVID-19 cases }\end{array}$ \\
\hline $\begin{array}{ll}\text { L. li et.al } \\
{[100]}\end{array}$ & $\begin{array}{l}\text { Fully automatic frame- } \\
\text { work to detect COVID- } \\
19 \text { using CT images of } \\
\text { chest. }\end{array}$ & $\begin{array}{l}\text { - Developed a DL model, COVNet to detect COVID-19 } \\
\text { by extracted visual features from chest CT exams. } \\
\text { - Collected dataset consisted of } 4356 \text { chest CT exams } \\
\text { from } 3,322 \text { patients from six hospital. }\end{array}$ \\
\hline $\begin{array}{l}\text { L. Wang et.al } \\
38\end{array}$ & $\begin{array}{l}\text { Open source Chest X- } \\
\text { Ray Image dataset and a } \\
\text { deep CNN for Detection } \\
\text { of COVID-19 Cases. }\end{array}$ & $\begin{array}{l}\text { - Proposed a publicly available COVID-Net, a deep CNN } \\
\text { for the detection of COVID-19 cases from CXR images. } \\
\text { - COVIDx, an open access chest X-ray(CSR) dataset con- } \\
\text { sisting } 13,800 \text { CSR images across } 13,725 \text { patient. }\end{array}$ \\
\hline $\begin{array}{l}\text { S.J. Fong } \\
101\end{array}$ & $\begin{array}{l}\text { A forecasting model of } \\
\text { COVID- } 19 .\end{array}$ & $\begin{array}{l}\text { - A Composite Monte-Carlo simulation forecasting } \\
\text { model. } \\
\text { - A case study of using above simulation through deep } \\
\text { learning. }\end{array}$ \\
\hline S. Chae 102 & $\begin{array}{l}\text { Predicting infectious dis- } \\
\text { ease using DL and Big } \\
\text { data. }\end{array}$ & $\begin{array}{l}\text { - A study on DL and LSTM model over ARIMA model } \\
\text { to predict future infectious diseases. } \\
\text { - Proposed model tried to improve existing surveillance } \\
\text { systems to detect future infectious diseases. }\end{array}$ \\
\hline
\end{tabular}




\subsubsection{Analysis and Challenges}

From table 1 it could be drawn one conclusion that the majority of the works are for assisting radiologists to diagnose diseases. Some of are mentioned forecasting, fake news alert, etc, but more critical parts of this pandemic maybe addressed by this DL approach. Successfully apply DL in COVID-19 or any running pandemic has three main challenges. The first one is a shortage of reliable datasets. As data collection and validation are a time-consuming process as well as privacy issues also there whereas a pandemic or epidemic comes suddenly. The second one is the variety of data of a pandemic virus. This COVID-19 virus 'SARS-CoV-2' has mutating itself over different geographic regions, environments, and time [103, 104]. Therefore, the pandemic dataset collected from one region may not be work to drawn inference on the pandemic of other regions. The third one high computational resources required for a DL model whereas to cope with an outbreak IoT or Edge Device (ED) are useful for many purposes [16]. Though these types of equipments have low computing resources.

In order to overcome such challenges, cleaver implementation of relevant AI strategies is required. For the first two challenges, DTL or few shot learning and GAN [29] could be a possible approach towards possible solutions. DTL has described in section 4.2 whereas details about GAN are out of the scope of this article. The third challenge could be mitigated using Cloud Computing, Fog Computing, and Edge Computing [105]. However, for Cloud or even Fog Computing latency and data security \& privacy could be a problem. Therefore, Edge Computing could be effective for the third challenge, which has described in section 4.3 .

\subsection{Deep Transfer Learning to Mitigate Pandemic}

\subsubsection{Potentiality}

Section 2.2 has described about Deep Transfer Learning (DTL) in general. In this sections, how DTL could help to mitigate COVID-19 like pandemics is described. As mentioned, sufficient datasets of COVID-19 or any running pandemic are difficult to develop in a short period of time. Therefore, to exploit the benefit of DL to cope with COVID-19 or other pandemics are a bit challenging. Therefore, DTL could be effective in this case. As through DTL a DL model could be trained using a large scale benchmark dataset and learned features could be used in the domain of COVID-19 [54]. Many researchers are trying hard to use this DTL in the domain COVID-19 for many purposes. We have tried to summarize in table 2 some of the recent state-ofthe-art along with their main contribution towards mitigation of pandemics. As the number of peer-reviewed work is limited as this pandemic is new, so 
this table also has listed some pre-print works, which have tried to introduce some of the contributions in mitigating this current pandemic.

Table 2: Recent DTL based works to mitigate pandemics.

\begin{tabular}{|c|c|c|}
\hline $\begin{array}{l}\text { Reference } \\
\text { of Proposed } \\
\text { Works }\end{array}$ & $\begin{array}{l}\text { Dedicated tasks } \\
\text { of a pandemic }\end{array}$ & Main contributions \\
\hline $\begin{array}{l}\text { J. P. Cohen } \\
\text { et.al } 106\end{array}$ & $\begin{array}{l}\text { A severity score predic- } \\
\text { tion model for COVID- } \\
19 \text { pneumonia for frontal } \\
\text { chest X-ray images using } \\
\text { beside tool. }\end{array}$ & $\begin{array}{l}\text { - A DTL model that was pre-trained on large size non- } \\
\text { COVID-19 chest X-ray datasets for predicting COVID- } 19 \\
\text { peumonia. This study use pre-trained model predicts a } \\
\text { geographic extent score of range } 0-8 \text { with } 1.14 \text { MAE and } \\
\text { lung opacity score of range } 0-6 \text { with } 0.78 \text { MAE. } \\
\text { - A COVID- } 19 \text { chest image datset from a public COVID- } \\
19 \text { database were scored retrospectively by three blinded } \\
\text { experts. }\end{array}$ \\
\hline $\begin{array}{l}\text { S.Minaee } \\
\text { et.al } 107\end{array}$ & $\begin{array}{l}\text { Predicting } \\
\text { From COVID-19 } \\
\text { Images. }\end{array}$ & $\begin{array}{l}\text { - DTL on a subset of } 2000 \text { of } 5000 \text { radiograms was used to } \\
\text { train four popular CNN, including ResNet18, ResNet50, } \\
\text { SqueezeNet, and DenseNet-121, to identify COVID-19 } \\
\text { disease. } \\
\text { - Evaluated these trained models on the remaining } 3000 \\
\text { radiograms and achieved a sensitivity rate of } 97 \%(5 \%) \text {, } \\
\text { while a specificity rate of } 90 \% \text { approx. }\end{array}$ \\
\hline $\begin{array}{l}\text { S.Basu } \\
\text { [108 }\end{array}$ & $\begin{array}{l}\text { Screening COVID-19 us- } \\
\text { ing Chest X-Ray Images. }\end{array}$ & $\begin{array}{l}\text { - A domain extension transfer learning with pre-trained } \\
\text { deep CNN is tuned for classifying among four classes: nor- } \\
\text { mal, other diseases, pneumonia, and Covid } 19 \text {. } \\
\text { - A 5-fold cross-validation has experimented and overall } \\
\text { accuracy measured } 95.3 \% 0.02 \text {. }\end{array}$ \\
\hline $\begin{array}{l}\text { N.E.M Khal- } \\
\text { ifa et.al [109] }\end{array}$ & $\begin{array}{l}\text { An Experimental Case } \\
\text { on a limited COVID-19 } \\
\text { chest X-Ray dataset. }\end{array}$ & $\begin{array}{l}\text { - A study on neutrosophic and deep transfer learning } \\
\text { models on limited COVID-19 chest X-Ray dataset. } \\
\text { - They first converted grayscale X-ray images into } \\
\text { neutrosophic images then applied pre-trained Alexnet, } \\
\text { Googlenet, and Restnet } 18 \text { to classify four classes: } \\
\text { COVID-19, Normal, Pneumonia bacterial, and Pneumo- } \\
\text { nia virus. }\end{array}$ \\
\hline $\begin{array}{l}\text { B.R. Beck } \\
\text { et.al } 110 \text { ] }\end{array}$ & $\begin{array}{l}\text { Predicting commercially } \\
\text { available antiviral drugs } \\
\text { that may act on SARS- } \\
\text { CoV-2 through the DL } \\
\text { model and a drug-target } \\
\text { interaction. }\end{array}$ & $\begin{array}{l}\text { - Drug-target interaction model called MT-DTI to rec- } \\
\text { ognize commercially available drugs that could act on } \\
\text { SARS-CoV-2. } \\
\text { - Proposed a list of antiviral drugs identified by this MT- } \\
\text { DTI model. }\end{array}$ \\
\hline $\begin{array}{l}\text { A. Narin et.al } \\
\text { [111] }\end{array}$ & $\begin{array}{l}\text { Automatic Detection of } \\
\text { COVID-19. }\end{array}$ & $\begin{array}{l}\text { - Three different CNN (ResNet50, InceptionV3, and } \\
\text { Inception-ResNetV2)-based models for the detection of } \\
\text { COVID-19 pneumonia infection using X-ray radiography. } \\
\text { - Proposed pre-trained ResNet50 has given the best result } \\
\text { among these three. }\end{array}$ \\
\hline $\begin{array}{l}\text { I.D. Apos- } \\
\text { tolopoulos } \\
\text { et.al 54 }\end{array}$ & $\begin{array}{l}\text { Performance evaluation } \\
\text { of state-of-the-art CNN } \\
\text { architectures through } \\
\text { TL over medical image } \\
\text { classification. }\end{array}$ & $\begin{array}{l}\text { - Suggested DL with X-ray imaging may extract signifi- } \\
\text { cant bio-markers related to the COVID-19 disease. } \\
\text { - A dataset of } 1427 \text { X-ray images consisting of } 224 \text { im- } \\
\text { ages of Covid-19 disease, } 700 \text { images of common bacterial } \\
\text { pneumonia, and } 504 \text { images of normal conditions. }\end{array}$ \\
\hline $\begin{array}{l}\text { B. Subirana } \\
\text { et.al } 112 \text {. }\end{array}$ & $\begin{array}{l}\text { New crowdsource AI } \\
\text { approach to support } \\
\text { health care dealing with } \\
\text { COVID-19. }\end{array}$ & $\begin{array}{l}\text { - A proposed label works on recognition of cough sound } \\
\text { recording by phone as a diagnostic test for COVID- } 19 \text {. }\end{array}$ \\
\hline $\begin{array}{l}\text { N.E.M. Khal- } \\
\text { ifa et.al [113] }\end{array}$ & $\begin{array}{l}\text { Detection } \\
\text { COVID-19 } \\
\text { using GAN and TL } \\
\text { method. }\end{array}$ & $\begin{array}{l}\text { - A combination of GAN and DTL models for enhancing } \\
\text { testing accuracy. } \\
\text { - Their ResNet18-based combined model achieved state- } \\
\text { of-the-art accuracy in a chest x-ray dataset. }\end{array}$ \\
\hline
\end{tabular}




\subsubsection{Analysis and Challenges}

DTL does task adaption that is very necessary for analyzing, diagnosing as well as mitigating COVID-19 like pandemics. The number of studies is not large; in addition most of the existing studies and experiments on COVID-19 were applied for chest image analysis as cited in table 2. Only a few among them are proposed for target drug interaction, cough sound classification, etc. Lots of work could be done to mitigate this pandemic such as Intensive Care Unit(ICU) Monitoring, Patient Care, Hygienic Practice Monitoring, Wearing Personal Protective Equipment(PPE) Monitoring, Monitoring Systematic Social Distancing, Automatic fever detection, rumor detection, economical and social impact, etc. Most of these works could be easier when AI is cooperating and forming such a model along with IoT or ED 14. Some issues could be solved by EC as described in section 4.3. Though a better system could be delivered when the most suitable algorithm applied on EC. One possibility of archiving this when DTL implemented alongside with EC which as conceptually describes in section 5 .

\subsection{Edge Computing to Mitigate Pandemic}

\subsubsection{Potentiality}

Edge or IoT devices-based sophisticated equipments such as smart medical equipment, webcam, drone, wearable sensors, etc are very useful in a pandemic like situations [114]. As mentioned in section 2.3, edge computing brings the computation to near edge devices. It reduces latency, security \& privacy issue, etc. Therefore, this computing paradigm will be very effective to mitigate a pandemic situation [115]. The researchers from all over the world are trying hard to bring this along with other AI techniques to mitigate current COVID-19 pandemic [16, 116]. So far only a limited number of studies have investigated the use of EC in obtain an efficient and effective mitigation system of COVID-19. This subsection tried to bring some potentiality and scopes which shall help to mitigate COVID-19 like pandemics. Table 3 has mentioned some EC based studies on COVID-19 and related healthcare.

\subsubsection{Analysis and Challenges}

EC works on site, so, many benefits could draw from EC with IoT or ED. Nevertheless as mentioned IoT or ED has limited computing resources. Therefore, to get the benefit of modern AI algorithm such as DL it is still challenging. To cope with these challenges researchers from all over the world are working hard to propose many ideas [69, 123, 18]. But so far only a few studies on EC in pandemic are proposed in limited areas of application as mentioned in table 3. Assisting many critical COVID-19 related tasks such 
Table 3: Recent EC based works to mitigate pandemics.

\begin{tabular}{|c|c|c|}
\hline $\begin{array}{l}\text { Reference } \\
\text { of Proposed } \\
\text { Works }\end{array}$ & $\begin{array}{l}\text { Dedicated task } \\
\text { related to a pandemic } \\
\text { or healthcare }\end{array}$ & Main contributions \\
\hline $\begin{array}{l}\text { A.Sufian et.al } \\
{[14]}\end{array}$ & $\begin{array}{l}\text { EC based model to stop } \\
\text { spread COVID-19 }\end{array}$ & $\begin{array}{l}\text { - An EC based ICU, Critical Areas monitoring model. } \\
\text { - Proposed DL and Computer Vision-based surveillance } \\
\text { model. }\end{array}$ \\
\hline $\begin{array}{l}\text { C.Hegde et.al } \\
{[117]}\end{array}$ & $\begin{array}{l}\text { An open-source EC for } \\
\text { clinical screening sys- } \\
\text { tem. }\end{array}$ & $\begin{array}{l}\text { - Fever and Cyanosis detection using visible and far- } \\
\text { infrared cameras emergency department. } \\
\text { - This image segmentation-based EC uses open source. } \\
\text { hardwares. }\end{array}$ \\
\hline $\begin{array}{l}\text { A.A.Abdellatif } \\
\text { et.al 118 }\end{array}$ & $\begin{array}{l}\text { Data and application- } \\
\text { specific energy-efficient } \\
\text { smart health systems }\end{array}$ & $\begin{array}{l}\text { - An optimizes medical data transmission from edge } \\
\text { nodes to the healthcare provider with energy efficiency } \\
\text { and quality-of-service. } \\
\text { - Managing a heterogeneous wireless network through EC } \\
\text { to provide fast emergency response. }\end{array}$ \\
\hline $\begin{array}{l}\text { A.H. Sudhro } \\
\text { et.al } 119\end{array}$ & $\begin{array}{l}\text { QoS optimization in } \\
\text { medical healthcare } \\
\text { applications. }\end{array}$ & $\begin{array}{l}\text { - A window-based Rate Control Algorithm to QoS in mo- } \\
\text { bile EC. } \\
\text { - A framework for Mobile EC based Medical Applications. }\end{array}$ \\
\hline $\begin{array}{l}\text { M.Chen et.al } \\
{[120]}\end{array}$ & $\begin{array}{l}\text { Smart Healthcare Sys- } \\
\text { tem. }\end{array}$ & $\begin{array}{l}\text { - Edge cognitive computing-based smart healthcare } \\
\text { mechanism to dynamic resource allocation in healthcare. }\end{array}$ \\
\hline $\begin{array}{l}\text { P.Pace et.al } \\
121\end{array}$ & $\begin{array}{l}\text { Efficient Applications } \\
\text { for Healthcare Industry } \\
4.0\end{array}$ & $\begin{array}{l}\text { - Proposed BodyEdge, an architecture suited for human- } \\
\text { centric applications in context of the emerging healthcare } \\
\text { industry. } \\
\text { - A tiny mobile client module with EC for better health } \\
\text { service. }\end{array}$ \\
\hline $\begin{array}{l}\text { H.Zhang et.al } \\
122\end{array}$ & $\begin{array}{l}\text { Smart Hospitals Using } \\
\text { Narrowband-IoT. }\end{array}$ & $\begin{array}{l}\text { - An architecture to connect intelligent things in smart } \\
\text { hospitals based on Narrowband IoT. } \\
\text { - Smart hospital by connecting intelligent with low la- } \\
\text { tency. }\end{array}$ \\
\hline
\end{tabular}

as remote sensing-based COVID-19 patient monitoring, Hygienic practice monitoring, systematic social distancing monitoring in a crowded area, etc could be done through EC [14]. This article brings a conceptual model of EC with DTL in section 5 as a future scope to cope with such challenges.

\subsection{Dataset to Mitigate Pandemic}

\subsubsection{Potentiality}

Data is the fuel of a modern computing. Whether it is medical field or retailer market, in every field data are the most precious things. Recent AI techniques are mostly follow data driven approaches [124, 125]. DL or DTL based algorithms almost fully depend on the dataset. Therefore, to cope with a pandemic, data is one of the driving forces. For a pandemic as COVID-19, the dataset could be chest X-ray, CT images, pathological images, geographical region based spreading patterns, seasonal behavior, regional mortality rates, impact on the economy, etc. [126. In table 4 some available datasets related to COVID-19 like pandemics are mentioned with brief description. 
Table 4: Some Dataset for COVID-19 like pandemics.

\begin{tabular}{|c|c|}
\hline $\begin{array}{l}\text { Name of dataset } \\
\text { and Reference }\end{array}$ & Brief description \\
\hline COVID-CT-Dataset [127]. & $\begin{array}{l}\text { - A publicly CT scan dataset consisting of } 275 \text { positives for COVID- } \\
19 \text { cases. }\end{array}$ \\
\hline $\begin{array}{l}\text { COVID-19 X-ray } \text { image } \\
\text { dataset with two different } \\
\text { combinations for Applying } \\
\text { with DTL models for ex- } \\
\text { perimenting with different } \\
\text { experimental setup. } 54\end{array}$ & $\begin{array}{l}\text { - One dataset of } 1427 \text { X-ray images consisting of } 224 \text { images of } \\
\text { Covid-19 disease, } 700 \text { images of common bacterial pneumonia, and } \\
504 \text { images of normal conditions. } \\
\text { - Another dataset of } 1442 \text { X-ray images consisting } 224 \text { images of } \\
\text { Covid-19 disease, } 714 \text { images of common bacterial pneumonia, and } \\
504 \text { images of normal conditions }\end{array}$ \\
\hline $\begin{array}{l}\text { COVID-19 Image Data Collec- } \\
\text { tion } 95 .\end{array}$ & $\begin{array}{l}\text { - It was a crowdsourcing hosting currently contains } 123 \text { frontal X- } \\
\text { rays images. }\end{array}$ \\
\hline Chest CT Images $[100]$ & $\begin{array}{l}\text { - A dataset consisted of } 4356 \text { chest CT exams from } 3,322 \text { patients. } \\
\text { - Data are collected from six hospitals of average age is } 4915 \text { years, } \\
\text { among them } 1838 \text { male patients. }\end{array}$ \\
\hline $\begin{array}{l}\text { Coronavirus Twitter Dataset } \\
\text { [128. }\end{array}$ & $\begin{array}{l}\text { - A multilingual COVID-19 Twitter dataset that has been continu- } \\
\text { ously collecting since Jan } 22,2020 \text {. } \\
\text { - It consists online conversation about COVID-19 to track scientific } \\
\text { misinformation, rumors, etc. }\end{array}$ \\
\hline COVIDx CXR Dataset [38. & $\begin{array}{l}\text { - This dataset consisting of } 13,800 \text { images of chest radiography across } \\
13,725 \text { patients. }\end{array}$ \\
\hline $\begin{array}{l}\text { Epidemiological } \quad \text { COVID-19 } \\
\text { data [129]. }\end{array}$ & $\begin{array}{l}\text { - Individual-level data from municipal, provincial, and national } \\
\text { health reports, as well as additional information from online reports. } \\
\text { - All data are geo-coded including where available, including symp- } \\
\text { toms, key dates, and travel history. }\end{array}$ \\
\hline H1N1 Fever Dataset [130]. & $\begin{array}{l}\text { - Two datasets collected at Narita International Airport during the } \\
\text { H1N1 pandemic } 2009 . \\
\text { - The first dataset only } 16 \text { candidates and the second one is } 1049 \\
\text { collected using infrared thermal scanners. }\end{array}$ \\
\hline $\begin{array}{l}\text { Registry data from the } 191820 \\
\text { pandemic. 131. }\end{array}$ & $\begin{array}{l}\text { - A high-quality vital registration data with mortality for the } 191820 \\
\text { pandemic from all countries. }\end{array}$ \\
\hline
\end{tabular}

\subsubsection{Analysis and Challenges}

As mentioned data is driving force to which bring the knowledge but it not easily available. Specially COVID-19 or a sudden pandemic, gathering data and arrange it in a knowledgeable form are not expected as an easy task. Although for COVID-19, many sectors are very active as a result many data sources are quickly oriented towards COVID-19 pandemic. Some data sources are listed in table 5 where COVID-19, as well as other pandemic data, are available, so, researchers may use them for many purposes. The main challenges are sufficient datasets especially machine-readable datasets in every affected sector are yet to be available. Therefore, that are the challenges for data-driven AL algorithms or models, hence existing studies on real data and analysis are few. Although some related datasets mentioned in table 4 are available but most of them are for clinical purposes. As said this novel coronavirus is behaving differently across geographic regions, different environments, etc. Therefore, data of one region may not be effective to enhance knowledge in other regions. Data privacy and security also are considered ones of the big issues. To this reason this article suggesting transfer learning 
Table 5: Some Data Sources for COVID-19 like pandemics.

\begin{tabular}{|c|c|}
\hline $\begin{array}{l}\text { Sources } \\
\text { and/or Reference }\end{array}$ & Brief description \\
\hline $\begin{array}{l}\text { World Health } \quad \text { Organiza- } \\
\text { tion(WHO) } 3]\end{array}$ & $\begin{array}{l}\text { - WHO leading this battle by providing each and every possible data } \\
\text { and information. } \\
\text { - Most of the data are unstructured so it bit challenging to feed into } \\
\text { an AI model. }\end{array}$ \\
\hline $\begin{array}{l}\text { Johns Hopkins University } \\
\text { is in the forefront to pro- } \\
\text { vide COVID-19 dataset } 132 \\
\text { through their portal: } \\
\text { https://coronavirus.jhu.edu }\end{array}$ & $\begin{array}{l}\text { - A machine-readable dataset that aggregates relevant data from } \\
\text { country-level governmental, academic sources, journalistic, etc. } \\
\text { - Some notable COVID-19 dataset are 'county-level time-series', } \\
\text { 'healthcare system-related metrics', 'climate', 'transit scores', 'hos- } \\
\text { pital', etc. }\end{array}$ \\
\hline $\begin{array}{l}\text { University of Oxford dataset } \\
\text { regarding COVID-19 at their } \\
\text { portal: } \\
\text { https://www.bsg.ox.ac.uk/news/ } \\
\text { coronavirus-research-blavatnik- } \\
\text { school. }\end{array}$ & $\begin{array}{l}\text { - Oxford Covid-19 Government Response Tracker (OxCGRT), an } \\
\text { index-based data indication which govt. taking what kind of policy. } \\
\text { - Several policy data of different govt. taken during pandemic in- } \\
\text { cluding education policy and their impact. }\end{array}$ \\
\hline $\begin{array}{l}\text { European Union provides an } \\
\text { open data portal: } \\
\text { https://www.europeandataportal.eu } \\
\text { /en/highlights/covid-19. }\end{array}$ & $\begin{array}{l}\text { - Open data and COVID-19: provide many dataset medical data, } \\
\text { spreading data, etc. } \\
\text { - An Interactive map are provided and by clicking region specific } \\
\text { dataset can be downloaded. }\end{array}$ \\
\hline $\begin{array}{l}\text { European Center for Dis- } \\
\text { ease Prevention and Con- } \\
\text { trol(ECDC): } \\
\text { https://qap.ecdc.europa.eu/public/ } \\
\text { extensions/COVID-19/COVID- } \\
\text { 19.html. }\end{array}$ & $\begin{array}{l}\text { - Many datasets about infectious diseases including COVID-19. } \\
\text { - Enhanced surveillance dataset including daily update dataset, } \\
\text { medical dataset, public health in communicable diseases }\end{array}$ \\
\hline $\begin{array}{l}\text { Google: } \\
\text { https://google.com/ covid19-map/. }\end{array}$ & $\begin{array}{l}\text { - Different statistical, numeral data including number active cases, } \\
\text { number of death, number of recovered. } \\
\text { - Provide COVID-19 interactive map in addition dedicated dataset } \\
\text { search engine that is also available. }\end{array}$ \\
\hline $\begin{array}{l}\text { GitHub: } \\
\text { https://github.com/open-covid- } \\
\text { 19/data. }\end{array}$ & $\begin{array}{l}\text { - An open repository where many datasets is stored. } \\
\text { - Many research projects stores their data and mentioned links to } \\
\text { their article, but they provide a link to see and access the COVID-19 } \\
\text { dataset. }\end{array}$ \\
\hline $\begin{array}{l}\text { Kaggle: } \\
\text { https://www.kaggle.com/c/covid19- } \\
\text { global-forecasting-week-\#. }\end{array}$ & $\begin{array}{l}\text { - An online community of data scientists and machine learning prac- } \\
\text { titioners } \\
\text { - Forecasting dataset and other COVID-19, or pandemic dataset } \\
\text { available. }\end{array}$ \\
\hline
\end{tabular}

approaches to be used in developing models for mitigating COVID-19 like pandemics or epidemics.

\section{A Precedent Pipeline of DTL over EC to Mitigate COVID-19.}

As mentioned 4.1.2, DL has some limitations to cope with the challenges of a pandemic whereas section 2.2 has described the task adaptability through DTL where data shortages are there. Section 2.3 mentioned the potentialities of EC where computing power is low. Therefore, the merging of these three computing models could be more effective in assisting the mitigation of pandemic situations. This combined model, that is, Deep Transfer Learning over Edge Computing (DTLEC) will take the power of DL through DTL as well as would be applicable in critical sectors by EC to cope with a sudden pandemic. There are some studies that exist in DTLEC as in [69] and 
some related work mentioned in section 3. However, these works are still in general concept or their proposed methods were targeted to some other application areas. As per literature studies, this idea has not been studied or experimented to mitigate COVID-19 pandemic. This section tried to present a precedent working pipeline of DTLEC to assist mitigation of pandemic or epidemic.

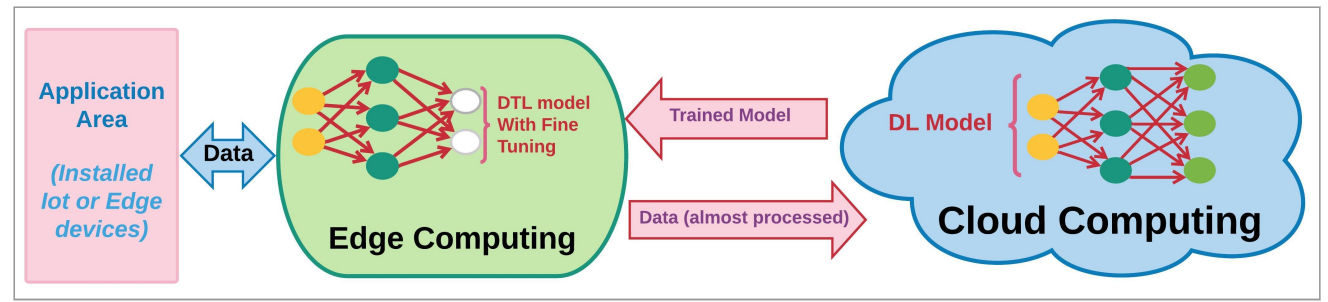

Figure 4: Proposed pipeline for DTL in EC.

\subsection{Model Description}

DTLEC model could be helpful in the healthcare sector, quarantine center, or other critical areas where an outbreak may arise. This methodology shall also use in remote health monitoring including elder people care at home. As in figure 4 edge or IoT devices that are set up in those areas may be embedded with EC, and then it could be connected with a cloud server. A state-of-the-art DL model shall train in GPU enabled cloud server by using a publicly available dataset for feature extraction. Then a pre-trained model (with extracted weight or features except for classification layer) shall push down to the edge devices. In edge devices required fine-tuning mechanism to be implemented into that model with some real data. In this way, the model may ready to work in some critical areas where outbreaks are affected such as hospitals, crowded places, and many more.

In figure 5 a typical current COVID-19 outbreaks situation and possible working model are shown. This figure illustrating the proposed framework to tackle the COVID-19 situation by using DTL in EC in both COVID19 patient care and management systematic social distancing. In the first scenario, we may use several healthcare sensors like blood pressure sensors, body temperature sensors, webcam, etc to sense the data about the running health condition of each patient. Then all of the collected data would be sent to the EC layer where a pre-trained DL based model will be used to process the captured data and making an inference out of it. If the generated report is a critical health condition then an automatic system alert message will be sent with all the details to the hospital control room and also to 


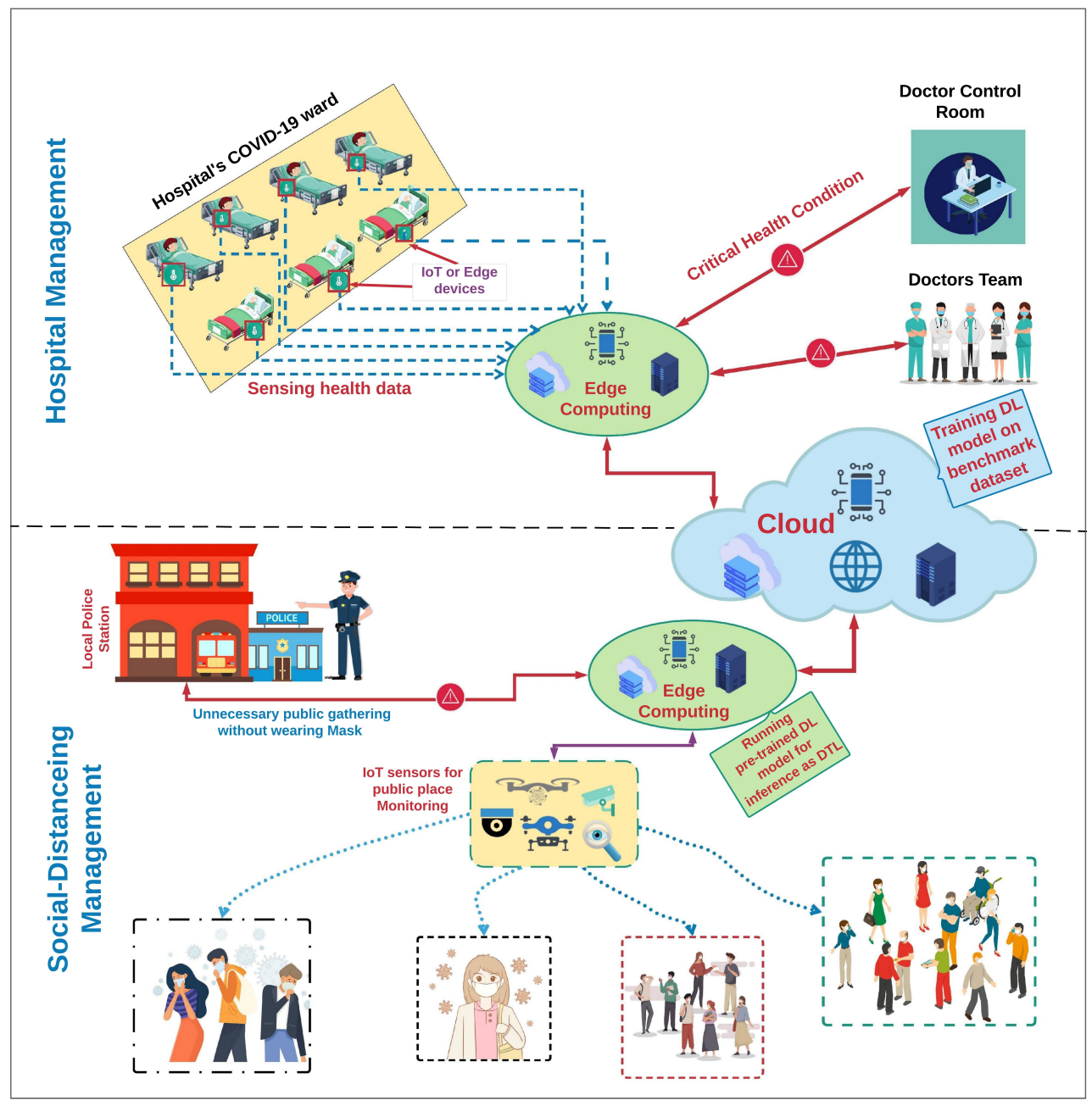

Figure 5: Framework for Edge Computing.

all the doctors of associated team. In the second scenario, several public place monitoring sensors (like a drone, CCTV, traffic cameras, etc) could be used to detect unnecessary illegal crowd with or without wearing personal protective equipment (PPE) with help from the DTLEC-based model. If the model finds any such activities then an automatic alert message will be sent with details to the nearby police station or any respective authority.

\subsection{Future Challenges}

This model may be successfully deployed in some critical sectors such as hospitals, airports, markets, emergency service areas, and those areas which are the primary hotshots for spreading pandemics. This methodology may use for remote health monitoring with further study. The model has to be 
work on real data to draw the inference. In order to make it successfully deployed, lots of collaborative work need to be done, which may face many challenges. Some challenges need to be addressed such as (i.) At first, IoT or Edge devices need to be connected with each other and a cloud server, hence a modified sensor type of networking protocol will be required. (ii.) EC through DTL need be implemented, for that appropriate pre-trained deep learning need be carefully selected after some studies. (iii.) For the transfer learning approach, using only EC is not sufficient, while the adoption of ECFog-Cloud combined model would be more useful. A deep learning model shall be trained at a cloud server using a benchmark dataset for feature extraction. After that pre-trained model will push down to the edge where limited re-training (or fine-tuning) shall be carried out to orient a few last layers for required inference. So, at least a small size pandemic dataset with ground truth label needs to be created. Here, the Fog server could work as a cluster. (iv.) Security and privacy issues of data need to be addressed. This inquires much more attention by researchers in analyzing numerous vulnerabilities that associated with such outbreak due to rumours and fake news. Besides, the privacy of captured data from multiple sources (things in IoT or individuals) will open a new research direction for the near coming future. (v.) A new simulation model may be required for experimental studies. These are a few of the other many challenges we can work for.

\section{Conclusion}

This article has tried to bring potentialities and challenges of Deep Transfer Learning, Edge Computing and their related issues to mitigate COVID-19 pandemic. It has also proposed a conceptual combined model with its scope and future challenges of working at critical sites and real data. As the running pandemic is new, so, there is a limited number of peer-reviewed studies and experimental results. Therefore, this systematic study article also considered some pre-print studies which are tried to make some contributions in mitigating running pandemic. The running pandemic definitely will be mitigated but there will be a leftover impact on global health, economics, education, etc. Therefore, mitigation of this pandemic as early as possible becomes necessary to restrict further worsen. In addition, every scientific community of the world needs to think wisely to get prepared to cope with such kind of crisis in a case similar outbreaks appear in the future. This article will definitely assist to the research community; especially deep transfer learning and edge computing to work further in developing many methodologies, tools and applications towards the mitigation of running pandemic or any future pandemic if that could arise. 


\section{References}

[1] H. A. Rothan, S. N. Byrareddy, The epidemiology and pathogenesis of coronavirus disease (covid-19) outbreak, Journal of autoimmunity (2020) 102433.

[2] Y. Jin, H. Yang, W. Ji, W. Wu, S. Chen, W. Zhang, G. Duan, Virology, epidemiology, pathogenesis, and control of covid-19, Viruses 12 (4) (2020) 372.

[3] W. H. Organization, et al., Coronavirus disease 2019 (covid-19): situation report, 74.

[4] C. Sohrabi, Z. Alsafi, N. ONeill, M. Khan, A. Kerwan, A. Al-Jabir, C. Iosifidis, R. Agha, World health organization declares global emergency: A review of the 2019 novel coronavirus (covid-19), International Journal of Surgery.

[5] E. D. Kilbourne, Influenza pandemics of the 20th century, Emerging infectious diseases 12 (1) (2006) 9.

[6] Y.-C. Hsieh, T.-Z. Wu, D.-P. Liu, P.-L. Shao, L.-Y. Chang, C.-Y. Lu, C.-Y. Lee, F.-Y. Huang, L.-M. Huang, Influenza pandemics: past, present and future, Journal of the Formosan Medical Association 105 (1) (2006) 1-6.

[7] W. H. Organization, et al., Rational use of personal protective equipment for coronavirus disease ( covid-19) and considerations during severe shortages: interim guidance, 6 april 2020, Tech. rep., World Health Organization (2020).

[8] P. Daszak, K. J. Olival, H. Li, A strategy to prevent future pandemics similar to the 2019-ncov outbreak (2020).

[9] A. S. Fauci, H. C. Lane, R. R. Redfield, Covid-19navigating the uncharted (2020).

[10] D. Fanelli, F. Piazza, Analysis and forecast of covid-19 spreading in china, italy and france, Chaos, Solitons \& Fractals 134 (2020) 109761.

[11] A. J. Kucharski, T. W. Russell, C. Diamond, Y. Liu, J. Edmunds, S. Funk, R. M. Eggo, F. Sun, M. Jit, J. D. Munday, et al., Early dynamics of transmission and control of covid-19: a mathematical modelling study, The lancet infectious diseases.

[12] Y. LeCun, Y. Bengio, G. Hinton, Deep learning, nature 521 (7553) (2015) 436-444.

[13] F. Shi, J. Wang, J. Shi, Z. Wu, Q. Wang, Z. Tang, K. He, Y. Shi, D. Shen, Review of artificial intelligence techniques in imaging data acquisition, segmentation and diagnosis for covid-19, arXiv preprint arXiv:2004.02731.

[14] A. Sufian, D. S. Jat, A. Banerjee, Insights of artificial intelligence to stop spread of covid-19, in: Big Data Analytics and Artificial Intelligence against COVID-19: Inovative Vision and Approach.

[15] C. Tan, F. Sun, T. Kong, W. Zhang, C. Yang, C. Liu, A survey on deep transfer learning, in: International conference on artificial neural networks, Springer, 2018, pp. 270-279. 
[16] D. S. W. Ting, L. Carin, V. Dzau, T. Y. Wong, Digital technology and covid-19, Nature Medicine (2020) 1-3.

[17] W. Z. Khan, E. Ahmed, S. Hakak, I. Yaqoob, A. Ahmed, Edge computing: A survey, Future Generation Computer Systems 97 (2019) 219-235.

[18] X. Wang, Y. Han, V. C. Leung, D. Niyato, X. Yan, X. Chen, Convergence of edge computing and deep learning: A comprehensive survey, IEEE Communications Surveys \& Tutorials.

[19] L. Nanni, S. Ghidoni, S. Brahnam, Handcrafted vs. non-handcrafted features for computer vision classification, Pattern Recognition 71 (2017) 158-172.

[20] I. Goodfellow, Y. Bengio, A. Courville, Deep learning, MIT press, 2016.

[21] G. Litjens, T. Kooi, B. E. Bejnordi, A. A. A. Setio, F. Ciompi, M. Ghafoorian, J. A. Van Der Laak, B. Van Ginneken, C. I. Sánchez, A survey on deep learning in medical image analysis, Medical image analysis 42 (2017) 60-88.

[22] X. Yao, Evolving artificial neural networks, Proceedings of the IEEE 87 (9) (1999) $1423-1447$.

[23] D. E. Rumelhart, G. E. Hinton, R. J. Williams, Learning internal representations by error propagation, Tech. rep., California Univ San Diego La Jolla Inst for Cognitive Science (1985).

[24] A. C. Wilson, R. Roelofs, M. Stern, N. Srebro, B. Recht, The marginal value of adaptive gradient methods in machine learning, in: Advances in Neural Information Processing Systems, 2017, pp. 4148-4158.

[25] Y. LeCun, Y. Bengio, et al., Convolutional networks for images, speech, and time series, The handbook of brain theory and neural networks 3361 (10) (1995) 1995.

[26] A. Ghosh, A. Sufian, F. Sultana, A. Chakrabarti, D. De, Fundamental concepts of convolutional neural network, in: Recent Trends and Advances in Artificial Intelligence and Internet of Things, Springer, 2020, pp. 519-567.

[27] D. P. Mandic, J. Chambers, Recurrent neural networks for prediction: learning algorithms, architectures and stability, John Wiley \& Sons, Inc., 2001.

[28] H. Sak, A. W. Senior, F. Beaufays, Long short-term memory recurrent neural network architectures for large scale acoustic modeling.

[29] I. Goodfellow, J. Pouget-Abadie, M. Mirza, B. Xu, D. Warde-Farley, S. Ozair, A. Courville, Y. Bengio, Generative adversarial nets, in: Advances in neural information processing systems, 2014, pp. 2672-2680.

[30] A. Krizhevsky, I. Sutskever, G. E. Hinton, Imagenet classification with deep convolutional neural networks, in: Advances in neural information processing systems, 2012, pp. 1097-1105. 
[31] M. D. Zeiler, R. Fergus, Visualizing and understanding convolutional networks, in: European conference on computer vision, Springer, 2014, pp. 818-833.

[32] K. Simonyan, A. Zisserman, Very deep convolutional networks for large-scale image recognition, arXiv preprint arXiv:1409.1556.

[33] C. Szegedy, W. Liu, Y. Jia, P. Sermanet, S. Reed, D. Anguelov, D. Erhan, V. Vanhoucke, A. Rabinovich, Going deeper with convolutions, in: Proceedings of the IEEE conference on computer vision and pattern recognition, 2015, pp. 1-9.

[34] K. He, X. Zhang, S. Ren, J. Sun, Deep residual learning for image recognition, in: Proceedings of the IEEE conference on computer vision and pattern recognition, 2016, pp. 770-778.

[35] G. Huang, Z. Liu, L. Van Der Maaten, K. Q. Weinberger, Densely connected convolutional networks, in: Proceedings of the IEEE conference on computer vision and pattern recognition, 2017, pp. 4700-4708.

[36] F. Sultana, A. Sufian, P. Dutta, Advancements in image classification using convolutional neural network, in: 2018 Fourth International Conference on Research in Computational Intelligence and Communication Networks (ICRCICN), IEEE, 2018, pp. $122-129$.

[37] S. Wang, B. Kang, J. Ma, X. Zeng, M. Xiao, J. Guo, M. Cai, J. Yang, Y. Li, X. Meng, et al., A deep learning algorithm using ct images to screen for corona virus disease (covid-19), medRxiv.

[38] L. Wang, A. Wong, Covid-net: A tailored deep convolutional neural network design for detection of covid-19 cases from chest radiography images, arXiv preprint arXiv:2003.09871.

[39] S. J. Pan, Q. Yang, A survey on transfer learning, IEEE Transactions on knowledge and data engineering 22 (10) (2009) 1345-1359.

[40] A. R. Zamir, A. Sax, W. Shen, L. J. Guibas, J. Malik, S. Savarese, Taskonomy: Disentangling task transfer learning, in: Proceedings of the IEEE Conference on Computer Vision and Pattern Recognition, 2018, pp. 3712-3722.

[41] R. Altman, Artificial intelligence (ai) systems for interpreting complex medical datasets, Clinical Pharmacology \& Therapeutics 101 (5) (2017) 585-586.

[42] Y. LeCun, 1.1 deep learning hardware: Past, present, and future, in: 2019 IEEE International Solid- State Circuits Conference - (ISSCC), 2019, pp. 12-19.

[43] S. Mittal, S. Vaishay, A survey of techniques for optimizing deep learning on gpus, Journal of Systems Architecture 99 (2019) 101635. doi:https://doi.org/10. 1016/j.sysarc.2019.101635

[44] M. Long, H. Zhu, J. Wang, M. I. Jordan, Deep transfer learning with joint adaptation networks, in: Proceedings of the 34th International Conference on Machine Learning-Volume 70, JMLR. org, 2017, pp. 2208-2217. 
[45] S. Koitka, C. M. Friedrich, Traditional feature engineering and deep learning approaches at medical classification task of imageclef 2016., in: CLEF (Working Notes), 2016, pp. 304-317.

[46] A. Kumar, J. Kim, D. Lyndon, M. Fulham, D. Feng, An ensemble of fine-tuned convolutional neural networks for medical image classification, IEEE Journal of Biomedical and Health Informatics 21 (1) (2017) 31-40.

[47] Y. Gao, K. M. Mosalam, Deep transfer learning for image-based structural damage recognition, Computer-Aided Civil and Infrastructure Engineering 33 (9) (2018) $748-768$.

[48] J. Deng, W. Dong, R. Socher, L. Li, Kai Li, Li Fei-Fei, Imagenet: A large-scale hierarchical image database, in: 2009 IEEE Conference on Computer Vision and Pattern Recognition, 2009, pp. 248-255.

[49] M. Talo, U. B. Baloglu, Ö. Yıldırım, U. R. Acharya, Application of deep transfer learning for automated brain abnormality classification using mr images, Cognitive Systems Research 54 (2019) 176-188.

[50] T. Han, C. Liu, W. Yang, D. Jiang, Deep transfer network with joint distribution adaptation: A new intelligent fault diagnosis framework for industry application, ISA transactions 97 (2020) 269-281.

[51] T. V. Phan, S. Sultana, T. G. Nguyen, T. Bauschert, $q$-transfer: A novel framework for efficient deep transfer learning in networking, in: 2020 International Conference on Artificial Intelligence in Information and Communication (ICAIIC), IEEE, 2020, pp. 146-151.

[52] T. S. Santosh, R. Parmar, H. Anand, K. Srikanth, M. Saritha, A review of salivary diagnostics and its potential implication in detection of covid-19, Cureus 12 (4).

[53] M. Loey, F. Smarandache, N. E. M. Khalifa, Within the lack of covid-19 benchmark dataset: A novel gan with deep transfer learning for corona-virus detection in chest $\mathrm{x}$-ray images $12(4)$.

[54] I. D. Apostolopoulos, T. A. Mpesiana, Covid-19: automatic detection from x-ray images utilizing transfer learning with convolutional neural networks, Physical and Engineering Sciences in Medicine (2020) 1.

[55] I. Baldini, P. Castro, K. Chang, P. Cheng, S. Fink, V. Ishakian, N. Mitchell, V. Muthusamy, R. Rabbah, A. Slominski, et al., Serverless computing: Current trends and open problems, in: Research Advances in Cloud Computing, Springer, 2017, pp. 1-20.

[56] M. Satyanarayanan, The emergence of edge computing, Computer 50 (1) (2017) 30-39.

[57] M. Garca-Valls, A. Dubey, V. Botti, Introducing the new paradigm of social dispersed computing: Applications, technologies and challenges, Journal of Systems Architecture 91 (2018) 83 - 102. doi :https://doi.org/10.1016/j.sysarc.2018. 05.007 
[58] R. Roman, J. Lopez, M. Mambo, Mobile edge computing, fog et al.: A survey and analysis of security threats and challenges, Future Generation Computer Systems 78 (2018) 680-698.

[59] W. Shi, J. Cao, Q. Zhang, Y. Li, L. Xu, Edge computing: Vision and challenges, IEEE internet of things journal 3 (5) (2016) 637-646.

[60] A. Yousefpour, C. Fung, T. Nguyen, K. Kadiyala, F. Jalali, A. Niakanlahiji, J. Kong, J. P. Jue, All one needs to know about fog computing and related edge computing paradigms: A complete survey, Journal of Systems Architecture 98 (2019) 289 330. doi:https://doi.org/10.1016/j.sysarc.2019.02.009.

[61] X. Sun, N. Ansari, Edgeiot: Mobile edge computing for the internet of things, IEEE Communications Magazine 54 (12) (2016) 22-29.

[62] S. Yi, C. Li, Q. Li, A survey of fog computing: concepts, applications and issues, in: Proceedings of the 2015 workshop on mobile big data, 2015, pp. 37-42.

[63] F. Wang, J. Xu, X. Wang, S. Cui, Joint offloading and computing optimization in wireless powered mobile-edge computing systems, IEEE Transactions on Wireless Communications 17 (3) (2017) 1784-1797.

[64] W. Ding, Z. Huang, Z. Huang, L. Tian, H. Wang, S. Feng, Designing efficient accelerator of depthwise separable convolutional neural network on fpga, Journal of Systems Architecture 97 (2019) 278 - 286. doi:https://doi.org/10.1016/j.sysarc. 2018.12.008

[65] G. Premsankar, M. Di Francesco, T. Taleb, Edge computing for the internet of things: A case study, IEEE Internet of Things Journal 5 (2) (2018) 1275-1284.

[66] S. Wang, Y. Zhao, J. Xu, J. Yuan, C.-H. Hsu, Edge server placement in mobile edge computing, Journal of Parallel and Distributed Computing 127 (2019) 160-168.

[67] A. Ksentini, P. A. Frangoudis, Toward slicing-enabled multi-access edge computing in 5g, IEEE Network 34 (2) (2020) 99-105.

[68] J. Chen, X. Ran, Deep learning with edge computing: A review, Proceedings of the IEEE 107 (8) (2019) 1655-1674.

[69] H. Li, K. Ota, M. Dong, Learning iot in edge: Deep learning for the internet of things with edge computing, IEEE network 32 (1) (2018) 96-101.

[70] S. Mittal, A survey on optimized implementation of deep learning models on the nvidia jetson platform, Journal of Systems Architecture 97 (2019) 428 - 442. doi: https://doi.org/10.1016/j.sysarc.2019.01.011.

[71] Q. Chen, Z. Zheng, C. Hu, D. Wang, F. Liu, On-edge multi-task transfer learning: Model and practice with data-driven task allocation, IEEE Transactions on Parallel and Distributed Systems 31 (6) (2020) 1357-1371. 
[72] L. Valerio, A. Passarella, M. Conti, Accuracy vs. traffic trade-off of learning iot data patterns at the edge with hypothesis transfer learning, in: 2016 IEEE 2nd International Forum on Research and Technologies for Society and Industry Leveraging a better tomorrow (RTSI), IEEE, 2016, pp. 1-6.

[73] T. Hou, G. Feng, S. Qin, W. Jiang, Proactive content caching by exploiting transfer learning for mobile edge computing, International Journal of Communication Systems 31 (11) (2018) e3706.

[74] J. Wang, Z. Feng, Z. Chen, S. George, M. Bala, P. Pillai, S.-W. Yang, M. Satyanarayanan, Bandwidth-efficient live video analytics for drones via edge computing, in: 2018 IEEE/ACM Symposium on Edge Computing (SEC), IEEE, 2018, pp. 159173.

[75] R. Sharma, S. Biookaghazadeh, B. Li, M. Zhao, Are existing knowledge transfer techniques effective for deep learning with edge devices?, in: 2018 IEEE International Conference on Edge Computing (EDGE), IEEE, 2018, pp. 42-49.

[76] Q. Chen, Z. Zheng, C. Hu, D. Wang, F. Liu, Data-driven task allocation for multitask transfer learning on the edge, in: 2019 IEEE 39th International Conference on Distributed Computing Systems (ICDCS), IEEE, 2019, pp. 1040-1050.

[77] W. Sun, J. Liu, Y. Yue, Ai-enhanced offloading in edge computing: when machine learning meets industrial iot, IEEE Network 33 (5) (2019) 68-74.

[78] R.-T. Wu, A. Singla, M. R. Jahanshahi, E. Bertino, B. J. Ko, D. Verma, Pruning deep convolutional neural networks for efficient edge computing in condition assessment of infrastructures, Computer-Aided Civil and Infrastructure Engineering 34 (9) (2019) 774-789.

[79] H. Daga, P. K. Nicholson, A. Gavrilovska, D. Lugones, Cartel: A system for collaborative transfer learning at the edge, in: Proceedings of the ACM Symposium on Cloud Computing, 2019, pp. 25-37.

[80] Y. Chen, J. Wang, C. Yu, W. Gao, X. Qin, Fedhealth: A federated transfer learning framework for wearable healthcare, arXiv preprint arXiv:1907.09173.

[81] X. Zhang, Y. Wang, S. Lu, L. Liu, W. Shi, et al., Openei: An open framework for edge intelligence, in: 2019 IEEE 39th International Conference on Distributed Computing Systems (ICDCS), IEEE, 2019, pp. 1840-1851.

[82] C. She, R. Dong, Z. Gu, Z. Hou, Y. Li, W. Hardjawana, C. Yang, L. Song, B. Vucetic, Deep learning for ultra-reliable and low-latency communications in $6 \mathrm{~g}$ networks, arXiv preprint arXiv:2002.11045.

[83] G. Li, Y. Yao, J. Wu, X. Liu, X. Sheng, Q. Lin, A new load balancing strategy by task allocation in edge computing based on intermediary nodes, EURASIP Journal on Wireless Communications and Networking 2020 (1) (2020) 1-10.

[84] G. White, S. Clarke, Urban intelligence with deep edges, IEEE Access 8 (2020) 7518-7530. 
[85] J. Yang, H. Zou, S. Cao, Z. Chen, L. Xie, Mobileda: Towards edge domain adaptation, IEEE Internet of Things Journal.

[86] D. Preuveneers, I. Tsingenopoulos, W. Joosen, Resource usage and performance trade-offs for machine learning models in smart environments, Sensors 20 (4) (2020) 1176.

[87] G. Hinton, Deep learninga technology with the potential to transform health care, Jama 320 (11) (2018) 1101-1102.

[88] I. Tobore, J. Li, L. Yuhang, Y. Al-Handarish, A. Kandwal, Z. Nie, L. Wang, Deep learning intervention for health care challenges: Some biomedical domain considerations, JMIR mHealth and uHealth 7 (8) (2019) e11966.

[89] F. Ucar, D. Korkmaz, Covidiagnosis-net: Deep bayes-squeezenet based diagnostic of the coronavirus disease 2019 (covid-19) from x-ray images, Medical Hypotheses (2020) 109761.

[90] C. Butt, J. Gill, D. Chun, B. A. Babu, Deep learning system to screen coronavirus disease 2019 pneumonia, Applied Intelligence (2020) 1.

[91] S. Hu, Y. Gao, Z. Niu, Y. Jiang, L. Li, X. Xiao, M. Wang, E. F. Fang, W. MenpesSmith, J. Xia, et al., Weakly supervised deep learning for covid-19 infection detection and classification from ct images, arXiv preprint arXiv:2004.06689.

[92] T. Ozturk, M. Talo, E. A. Yildirim, U. Baloglu, O. Yildirim, U. R. Acharya, Automated detection of covid-19 cases using deep neural networks with x-ray images, Computers in Biology and Medicine.

[93] J. Redmon, A. Farhadi, Yolov3: An incremental improvement, arXiv preprint arXiv:1804.02767.

[94] E. Luz, P. L. Silva, R. Silva, G. Moreira, Towards an efficient deep learning model for covid-19 patterns detection in x-ray images, arXiv preprint arXiv:2004.05717.

[95] J. P. Cohen, P. Morrison, L. Dao, Covid-19 image data collection, arXiv preprint arXiv:2003.11597.

[96] M. Zhou, Y. Chen, D. Wang, Y. Xu, W. Yao, J. Huang, X. Jin, Z. Pan, J. Tan, L. Wang, et al., Improved deep learning model for differentiating novel coronavirus pneumonia and influenza pneumonia, medRxiv.

[97] A. Lopez-Rincon, A. Tonda, L. Mendoza-Maldonado, E. Claassen, J. Garssen, A. D. Kraneveld, Accurate identification of sars-cov-2 from viral genome sequences using deep learning, bioRxiv.

[98] O. Gozes, M. Frid-Adar, H. Greenspan, P. D. Browning, H. Zhang, W. Ji, A. Bernheim, E. Siegel, Rapid ai development cycle for the coronavirus (covid-19) pandemic: Initial results for automated detection \& patient monitoring using deep learning ct image analysis, arXiv preprint arXiv:2003.05037. 
[99] S. M. Ayyoubzadeh, S. M. Ayyoubzadeh, H. Zahedi, M. Ahmadi, S. R. N. Kalhori, Predicting covid-19 incidence through analysis of google trends data in iran: Data mining and deep learning pilot study, JMIR Public Health and Surveillance 6 (2) (2020) e18828.

[100] L. Li, L. Qin, Z. Xu, Y. Yin, X. Wang, B. Kong, J. Bai, Y. Lu, Z. Fang, Q. Song, et al., Artificial intelligence distinguishes covid-19 from community acquired pneumonia on chest ct, Radiology (2020) 200905.

[101] S. J. Fong, G. Li, N. Dey, R. G. Crespo, E. Herrera-Viedma, Composite monte carlo decision making under high uncertainty of novel coronavirus epidemic using hybridized deep learning and fuzzy rule induction, Applied Soft Computing (2020) 106282 .

[102] S. Chae, S. Kwon, D. Lee, Predicting infectious disease using deep learning and big data, International journal of environmental research and public health 15 (8) (2018) 1596.

[103] Z. Shen, Y. Xiao, L. Kang, W. Ma, L. Shi, L. Zhang, Z. Zhou, J. Yang, J. Zhong, D. Yang, et al., Genomic diversity of sars-cov-2 in coronavirus disease 2019 patients, Clinical Infectious Diseases.

[104] N. D. Grubaugh, M. E. Petrone, E. C. Holmes, We shouldnt worry when a virus mutates during disease outbreaks, Nature Microbiology 5 (4) (2020) 529-530.

[105] P. Escamilla-Ambrosio, A. Rodríguez-Mota, E. Aguirre-Anaya, R. Acosta-Bermejo, M. Salinas-Rosales, Distributing computing in the internet of things: cloud, fog and edge computing overview, in: NEO 2016, Springer, 2018, pp. 87-115.

[106] J. P. Cohen, L. Dao, P. Morrison, K. Roth, Y. Bengio, B. Shen, A. Abbasi, M. Hoshmand-Kochi, M. Ghassemi, H. Li, et al., Predicting covid-19 pneumonia severity on chest x-ray with deep learning, arXiv preprint arXiv:2005.11856.

[107] S. Minaee, R. Kafieh, M. Sonka, S. Yazdani, G. J. Soufi, Deep-covid: Predicting covid-19 from chest x-ray images using deep transfer learning, arXiv preprint arXiv:2004.09363.

[108] S. Basu, S. Mitra, Deep learning for screening covid-19 using chest x-ray images, arXiv preprint arXiv:2004.10507.

[109] N. E. M. Khalifa, F. Smarandache, M. Loey, A study of the neutrosophic set significance on deep transfer learning models: An experimental case on a limited covid-19 chest x-ray dataset, Symmetry.

[110] B. R. Beck, B. Shin, Y. Choi, S. Park, K. Kang, Predicting commercially available antiviral drugs that may act on the novel coronavirus (sars-cov-2) through a drugtarget interaction deep learning model, Computational and structural biotechnology journal.

[111] A. Narin, C. Kaya, Z. Pamuk, Automatic detection of coronavirus disease (covid19) using x-ray images and deep convolutional neural networks, arXiv preprint arXiv:2003.10849. 
[112] B. Subirana, F. Hueto, P. Rajasekaran, J. Laguarta, S. Puig, J. Malvehy, O. Mitja, A. Trilla, C. I. Moreno, J. F. M. Valle, et al., Hi sigma, do i have the coronavirus?: Call for a new artificial intelligence approach to support health care professionals dealing with the covid-19 pandemic, arXiv preprint arXiv:2004.06510.

[113] N. E. M. Khalifa, M. H. N. Taha, A. E. Hassanien, S. Elghamrawy, Detection of coronavirus (covid-19) associated pneumonia based on generative adversarial networks and a fine-tuned deep transfer learning model using chest x-ray dataset, arXiv preprint arXiv:2004.01184.

[114] H. Zhu, P. Podesva, X. Liu, H. Zhang, T. Teply, Y. Xu, H. Chang, A. Qian, Y. Lei, Y. Li, et al., Iot pcr for pandemic disease detection and its spread monitoring, Sensors and Actuators B: Chemical 303 (2020) 127098.

[115] M. Al-Zinati, Q. Al-Thebyan, Y. Jararweh, An agent-based self-organizing model for large-scale biosurveillance systems using mobile edge computing, Simulation Modelling Practice and Theory 93 (2019) 65-86.

[116] S. Wan, Z. Gu, Q. Ni, Cognitive computing and wireless communications on the edge for healthcare service robots, Computer Communications 149 (2020) 99-106.

[117] C. Hegde, P. B. Suresha, J. Zelko, Z. Jiang, R. Kamaleswaran, M. A. Reyna, G. D. Clifford, Autotriage-an open source edge computing raspberry pi-based clinical screening system, medRxiv.

[118] A. A. Abdellatif, A. Mohamed, C. F. Chiasserini, A. Erbad, M. Guizani, Edge computing for energy-efficient smart health systems: Data and application-specific approaches, in: Energy Efficiency of Medical Devices and Healthcare Applications, Elsevier, 2020, pp. 53-67.

[119] A. H. Sodhro, Z. Luo, A. K. Sangaiah, S. W. Baik, Mobile edge computing based qos optimization in medical healthcare applications, International Journal of Information Management 45 (2019) 308-318.

[120] M. Chen, W. Li, Y. Hao, Y. Qian, I. Humar, Edge cognitive computing based smart healthcare system, Future Generation Computer Systems 86 (2018) 403-411.

[121] P. Pace, G. Aloi, R. Gravina, G. Caliciuri, G. Fortino, A. Liotta, An edge-based architecture to support efficient applications for healthcare industry 4.0, IEEE Transactions on Industrial Informatics 15 (1) (2019) 481-489.

[122] H. Zhang, J. Li, B. Wen, Y. Xun, J. Liu, Connecting intelligent things in smart hospitals using nb-iot, IEEE Internet of Things Journal 5 (3) (2018) 1550-1560.

[123] M. Mohammadi, A. Al-Fuqaha, S. Sorour, M. Guizani, Deep learning for iot big data and streaming analytics: A survey, IEEE Communications Surveys \& Tutorials 20 (4) (2018) 2923-2960.

[124] F. Zhang, W. Li, Y. Zhang, Z. Feng, Data driven feature selection for machine learning algorithms in computer vision, IEEE Internet of Things Journal 5 (6) (2018) $4262-4272$. 
[125] X.-H. Li, C. C. Cao, Y. Shi, W. Bai, H. Gao, L. Qiu, C. Wang, Y. Gao, S. Zhang, $\mathrm{X}$. Xue, et al., A survey of data-driven and knowledge-aware explainable ai, IEEE Transactions on Knowledge and Data Engineering.

[126] T. Alamo, D. G. Reina, M. Mammarella, A. Abella, Open data resources for fighting covid-19, arXiv preprint arXiv:2004.06111.

[127] J. Zhao, Y. Zhang, X. He, P. Xie, Covid-ct-dataset: A ct scan dataset about covid19, arXiv preprint arXiv:2003.13865.

[128] E. Chen, K. Lerman, E. Ferrara, Covid-19: The first public coronavirus twitter dataset, arXiv preprint arXiv:2003.07372.

[129] B. Xu, B. Gutierrez, S. Mekaru, K. Sewalk, L. Goodwin, A. Loskill, E. L. Cohn, Y. Hswen, S. C. Hill, M. M. Cobo, et al., Epidemiological data from the covid-19 outbreak, real-time case information, Scientific data 7 (1) (2020) 1-6.

[130] H. Nishiura, K. Kamiya, Fever screening during the influenza (h1n1-2009) pandemic at narita international airport, japan, BMC infectious diseases 11 (1) (2011) 111.

[131] C. J. Murray, A. D. Lopez, B. Chin, D. Feehan, K. H. Hill, Estimation of potential global pandemic influenza mortality on the basis of vital registry data from the 191820 pandemic: a quantitative analysis, The Lancet 368 (9554) (2006) 2211-2218.

[132] B. D. Killeen, J. Y. Wu, K. Shah, A. Zapaishchykova, P. Nikutta, A. Tamhane, S. Chakraborty, J. Wei, T. Gao, M. Thies, et al., A county-level dataset for informing the united states' response to covid-19, arXiv preprint arXiv:2004.00756. 